\title{
A Study on Testing Techniques for Concrete-like Materials under Compressive Impact Loading
}

\author{
Han Zhao \\ Laboratoire de Mécanique des Solides, Ecole Polytechnique, 91128 Palaiseau, France
}

\begin{abstract}
An analysis of specific experimental problems encountered in the testing of concrete and concrete-like materials under impact loading, using the split Hopkinson pressure bar test (SHPB) or the so-called direct impact Hopkinson bar test (DIHB), is presented in this paper. On the one hand, in data processing, it is shown that recent improvements such as wave dispersion correction and exact time shifting are indispensable to obtain accurate measurements in those tests. On the other hand, the conventional analyses of those two kinds of tests are investigated with the aid of transient numerical simulations. It is concluded that only one of the conventionally used formula offers a good estimate of stress-strain curve for concrete-like materials. It is also illustrated that the usual assumptions of DIHB analysis are not reliable for the concrete-like specimen. An inverse approach is nevertheless possible to give more accurate results.
\end{abstract}

Keywords: testing, impact loads, concrete, data processing, split Hopkinson bar test, strain rate.

\section{INTRODUCTION}

The behaviour of concrete and concrete-like materials under impact loading is involved in the study of safety requirements of structures in an extreme situation such as earthquakes, accidental impacts or explosions. The early experimental data of those materials under dynamic loading are obtained with pendulum tests, drop weight tests, etc. A review of those works is given by Green. ${ }^{1}$ However, the 'state of the art' report of RILEM committee ${ }^{2}$ has given a comparison of results obtained from different loading devices, showing important gaps among different techniques. This is not only because of the heterogeneous nature of concrete-like materials or of different specimen dimensions. It is also because of the unavoidable imprecision of those loading and measuring devices.

The technique using split bars, which offers an accurate result under impact loading, has then been applied to the testing of those concrete-like materials. The use of the compressive Split Hopkinson Bar (SHB) to determine the rate sensitivity of concrete has been reported in recent works. ${ }^{3,4}$ The less frequent application of the tensile SHB arrangement to concrete specimens has been also reported. ${ }^{5}$ The direct impact Hopkinson bar test (DIHB) is used to perform the tests at medium strain rates. ${ }^{6}$ There also exists the possibility to impose loading-unloading cycles at high strain rates. $^{7}$ As those techniques using bars are initially developed for testing metals, its application in the case of concrete-like materials raises some specific problems.

This paper presents a study of those specific problems. On the one hand, in order to ensure the accuracy of experimental devices, measuring imprecision due to the wave dispersion in split bars and due to the error in the time shift is discussed and an optimal data processing is proposed. On the other hand, the analysis of the measured data (the forces and the velocities at the faces of specimen) is reconsidered. Trans- 


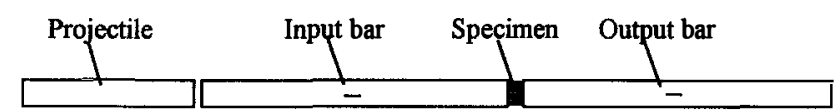

Fig. 1. Split Hopkinson pressure bar (SHPB) test.

ient numerical simulations of concrete specimens are performed with a rate-sensitive crack model. This permits a careful investigation of the accuracy of the stress-strain relation obtained by the conventional analysis for those tests on concrete.

\section{CONCRETE TESTING USING SPLIT BARS, OPTIMAL DATA PROCESSING}

\section{SHPB and DIHB tests}

The Split Hopkinson Pressure Bar (SHPB), or Kolsky's apparatus ${ }^{8}$ is somehow a universal experimental technique in the study of the constitutive law of materials at high strain rates. A typical SHPB set-up is outlined in Fig. 1. It is composed of the long input and output bars with a short specimen placed between them. The impact of the projectile at the free end of the input bar develops a compressive longitudinal incident wave $\varepsilon_{i}(t)$. Once it arrives at the bar specimen interface, a reflected wave $\varepsilon_{\mathrm{r}}(t)$ is developed in the input bar, whereas a transmitted wave $\varepsilon_{\mathrm{t}}(t)$ is developed in the output bar. When the waves are known at bar-specimen interfaces, the forces and the velocities at both faces of the specimen are given by the following eqn (1).

$$
\begin{aligned}
& \mathrm{F}_{\text {input }}(t)=S_{\mathrm{B}} E\left(\varepsilon_{\mathrm{i}}(t)+\varepsilon_{\mathrm{r}}(t)\right) \\
& \mathrm{V}_{\text {input }}(t)=C_{0}\left(\varepsilon_{\mathrm{i}}(t)-\varepsilon_{\mathrm{r}}(t)\right) \\
& \mathrm{F}_{\text {output }}(t)=S_{\mathrm{B}} E \varepsilon_{\mathrm{t}}(t) \\
& \mathrm{V}_{\text {output }}(t)=C_{0} \varepsilon_{\mathrm{t}}(t)
\end{aligned}
$$

where $S_{\mathrm{B}}, E$ and $C_{0}$ are respectively the bars' cross-sectional area, Young's modulus, and elastic wave speed.

The use of an input bar implies a limitation of the maximum force for a given impact velocity because of the linear relation between stress and particular velocity for a wave. The SHPB then fails to smash the concrete specimen at medium strain rates. A current solution is to strike the specimen dirrectly. A scheme of the arrangement of such a so-called direct impact Hopkinson bar (DIHB) test is shown in

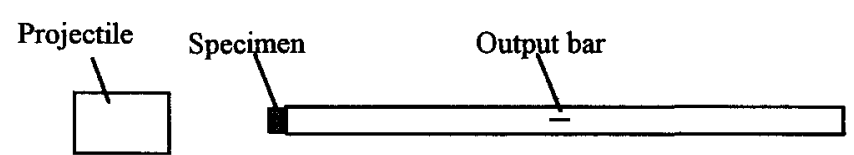

Fig. 2. Direct impact Hopkinson bar (DIHB) test.

Fig. 2. As measurements are only made at the output side, supplementary assumptions must be made for the input side. The usual method assumes an equilibrium state of the specimen so that the input force is equal to the output one. This method assumes also that the input velocity makes an instantaneous jump from zero to the measured initial velocity, $V_{0}$, of the projectile, and decelerates afterwards by the reaction force applied by the specimen on the projectile. $^{9-12}$

The velocity at the impacting face is then calculated by the following equation.

$$
\begin{gathered}
\mathrm{V}_{\text {input }}(t)=V_{0}-\frac{S_{\mathrm{B}} E}{S_{\mathrm{p}} \rho_{\mathrm{p}} C_{\mathrm{p}}}\left(\varepsilon_{\mathrm{t}}(t)+\sum_{n=1}^{N} 3 \varepsilon_{\mathrm{t}}(t-n T)\right) \\
\quad \text { if } N T \leq t<(N+1) T
\end{gathered}
$$

where $S_{\mathrm{p}}, \rho_{\mathrm{p}}, C_{\mathrm{p}}$ are respectively the cross-sectional area, the density and the wave velocity of the projectile. $N=\operatorname{int}(t / T), T$ being the characteristic time (time needed for the wave to perform a round trip in the projectile).

From those basic experimental data (forces and velocities at input and output sides), the conventional analysis assumes the axial uniformity of stress and strain fields in the specimen. An average strain and an average stress are then given by eqn (3).

$$
\begin{aligned}
& \dot{\varepsilon}_{\mathrm{s}}(t)=\frac{\mathrm{V}_{\text {output }}(t)-\mathrm{V}_{\text {input }}(t)}{l_{\mathrm{s}}}, \\
& \sigma_{\mathrm{s}}(t)=\frac{\mathrm{F}_{\text {input }}(t)}{S_{\mathrm{s}}}=\frac{\mathrm{F}_{\text {output }}(t)}{S_{\mathrm{s}}}
\end{aligned}
$$

where $l_{\mathrm{s}}, S_{\mathrm{s}}$ denote respectively the length and the cross-sectional area of the specimen.

\section{An optimal data processing for accurate measurements}

Experimental results reported in the literature on concrete-like materials show often relatively important dispersions, partly due to the measur- 
ing imprecision of experimental devices. As to the technique using bars, the main difficulty of the measurement lies in the fact that the three basic waves are measured at sections other than the bar-specimen interfaces. They have to be shifted from the strain gauges to the specimen faces, in time and distance. This shifting leads to two different perturbations. Firstly, waves change in their shapes (wave dispersion) on propagating along the bar. Secondly, one has to find the exact time shift for the three waves, so that the shifted waves at the bar-specimen interfaces correspond to the same time origin.

To avoid shifting errors, the correction of such so-called wave dispersion effect should be made. It consists of using an analytical solution of the harmonic wave propagation in an infinite cylindrical bar, ${ }^{3,13-15}$ which gives theoretical dispersion relation (wave velocities versus frequencies). An accurate determination of time shifts can be obtained by a method based on the simulation of the specimen response. ${ }^{16}$ In fact, one can simulate numerically, for an elastic specimen, the fictitious reflected and transmitted waves. The exact origins of those simulated waves are then known. If the real specimen is supposed elastic at the early stage of the test, the comparison between simulated and real waves at this early stage allows for the determination of their exact origins.

In order to show the influence of the data processing, a typical test on concrete using a $40 \mathrm{~mm}$ diameter bar is used as an example. Raw signals ( $1 \mathrm{~m}$ from bar-specimen interfaces) are compared with the signal after the dispersion correction (Fig. 3a). Even if the correction is not visually significant for the waves, it does for the final result (Fig. 3b). It is noted that the dispersion effect could be more important if a bar of a larger diameter $(80 \mathrm{~mm}$ bars for the larger aggregates) is used. As to the influence of the time shift, if an error of $3 \mu \mathrm{s}$ is deliberately made for the reflected wave, which is nearly unavoidable in an arbitrary visual determination (one can see the visual difference between the real and the broken lines is very small in Fig. 4a), the induced error in the average stress-strain curve is important (Fig. 4b).

Perturbations due to the measuring imprecision can be eliminated by the careful data processing. Indeed, a series of tests on a microconcrete (the part of dispersion due to the heterogeneity of specimens is limited) is performed to illustrate the testing reproducibility.
It is shown in Fig. 5 that both Young's modulus and the failure strength show only a small dispersion and the efficiency of the improved data processing is indirectly proved.

Application of those improvements in the data processing allows for more accurate results. They are particularly important for the accuracy in the range of small strain. Consequently, such an optimal data processing is indispensable not only for the concrete specimen but also for many materials, wherever the behaviour at small strains is the most desired (ceramics, ${ }^{17}$ composite, ${ }^{18}$ etc.).

\section{ANALYSIS OF MEASURED FORCES AND VELOCITIES IN CONCRETE TESTING}

Techniques using split bars provide, then, accurate dynamic measurements at the faces of the specimen (forces and velocities). However, those measurements do not provide immedia-

(a)

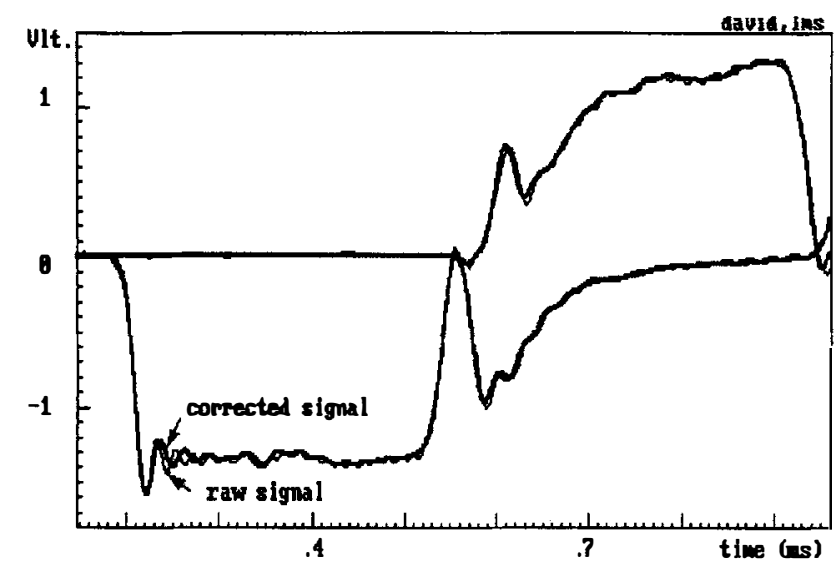

(b)

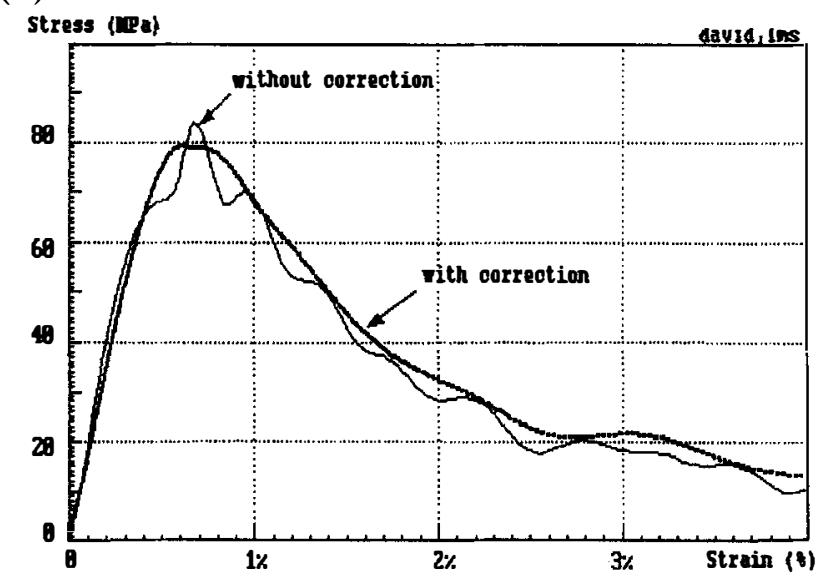

Fig. 3. (a)Visual difference of the wave dispersion correction. (b) Stress-strain curve, influence of the wave dispersion correction. 


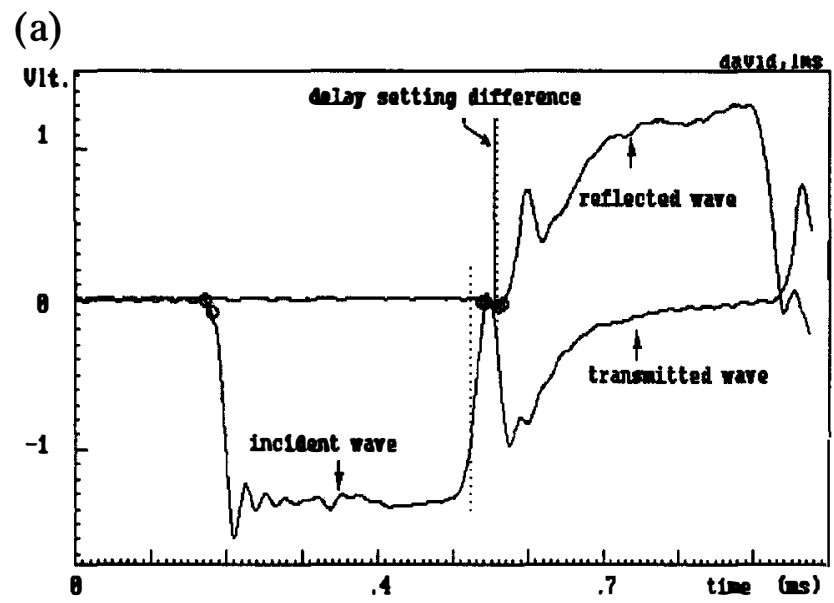

(b)



Fig. 4. (a) Visual difference of a $3 \mu$ s error in the delay setting. (b) Stress-strain curve, influence of $3 \mu \mathrm{s}$ error in the delay setting.

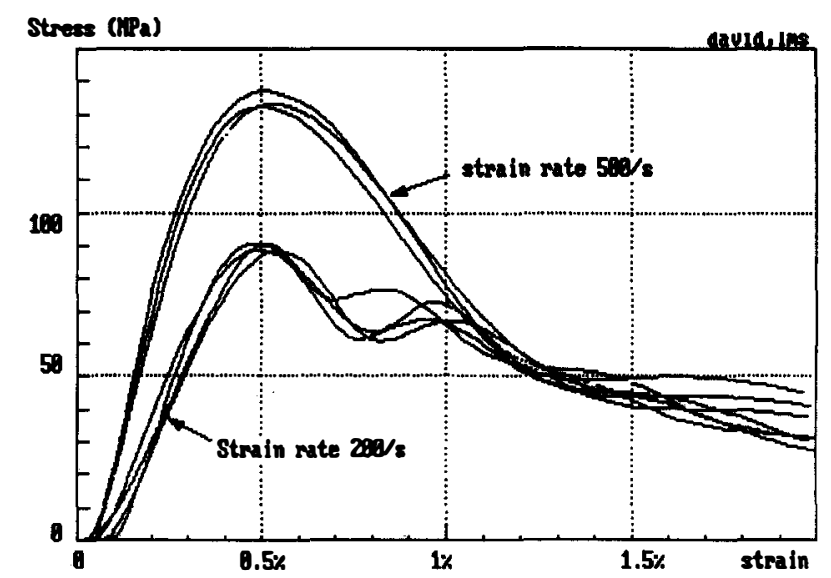

Fig. 5. Reproducibility of tests on micro-concrete specimen. tely the stress-strain relation of the specimen. At first, the stress-strain curves calculated by eqn (3) are correct only when stress and strain fields in the specimen are homogeneous. This is never true, because of transient effects in the specimen. For example, when the input side of the specimen is loaded, the output side remains at rest until the wave goes through the specimen. Furthermore, the average stress-strain curve is obtained under the one-dimensional assumption while the three-dimensional effects due to the radial inertia and to the friction could induce errors. In this paper, only the transient effect is discussed, knowing that threedimensional effect problems have been studied in past decades and corrections are proposed. ${ }^{19-21}$

In order to investigate if the non-homogeneity of the stress and strain fields introduce important error on the estimate of the stressstrain relation, one has to know both the behaviour of the specimen and the waves in the test. A natural method to do this is to simulate a test on a specimen of concrete-like materials. For this purpose, a constitutive law should be supposed for the specimen.

\section{Chosen constitutive law of concrete, numeric simulation using the method of characteristics}

A rate-dependant crack model is adopted to describe the rate-dependence and softening behaviour of a concrete-like material, because it is simple and successfully applied in the waveinvolved application. ${ }^{22}$ In this model, it is supposed that the behaviour is purely elastic when the total strain $\varepsilon$ is smaller than a crack threshold $\varepsilon_{\mathrm{s}}$.

$$
\varepsilon=\sigma / E \text { if } \varepsilon \leq \varepsilon_{\mathrm{s}}
$$

When the total strain is larger than the threshold, it is assumed to be the sum of the elastic strain $\varepsilon_{\mathrm{e}}$ and a so-called crack strain $\varepsilon_{\mathrm{cr}}$ due to the opening of the micro-cracks cumulating in the concrete. Using a simple linear dependence of the stress on the crack strain and the crack strain rate, a four-parameter model is formulated (eqn (4)).

$$
\varepsilon=\varepsilon_{e}+\varepsilon_{c r} \text { if } \varepsilon>\varepsilon_{s}
$$

with the two parts defined in the following:

$$
\dot{\varepsilon}_{\mathrm{e}}=\dot{\sigma} / E
$$




$$
\dot{\varepsilon}_{\mathrm{cr}}=\frac{\sigma-E \varepsilon_{\mathrm{s}}+k \varepsilon_{\mathrm{cr}}}{\eta}
$$

where $\sigma, \varepsilon, \varepsilon_{\mathrm{cr}}, \varepsilon_{\mathrm{s}}$ are respectively the stress, the total strain, the crack strain, and the crack threshold. $E, k, \eta$ are coefficients associated with elasticity, linear softening and viscosity.

The one-dimensional governing equations and the constitutive law are written as follows,

$$
\begin{gathered}
\frac{\partial \sigma(x, t)}{\partial x}=\rho \frac{\partial \mathrm{v}(x, t)}{\partial t} \\
\frac{\partial \varepsilon(x, t)}{\partial t}=\frac{\partial \mathrm{v}(x, t)}{\partial x}
\end{gathered}
$$

where $\sigma, \varepsilon, v$ are the stress, the strain, and the particle velocity in the specimen. $\rho$ is the mass density.

The governing equation (eqn (5)) is hyperbolic, and it can be then solved by the method of characteristics. Because of the form of constitutive law (eqn (4)), the characteristic network is composed of families of straight lines in this case. ${ }^{23}$ The three families of characteristic lines and the characteristic relation that must be satisfied along those lines are given in eqn (6).

(i) $\frac{\mathrm{d} x}{\mathrm{~d} t}=C_{0} \quad \mathrm{~d} \sigma=-\rho C_{0} \mathrm{~d} v-\rho C_{0}^{2} \dot{\varepsilon}_{\mathrm{cr}} \mathrm{d} t$

(ii) $\frac{\mathrm{d} x}{\mathrm{~d} t}=-C_{0} \quad \mathrm{~d} \sigma=-\rho C_{0} \mathrm{~d} v-\rho C_{0}^{2} \dot{\varepsilon}_{\mathrm{cr}} \mathrm{d} t$

(iii) $\mathrm{d} x=0 \quad \mathrm{~d} \varepsilon_{\mathrm{cr}}=\dot{\varepsilon}_{\mathrm{cr}} \mathrm{d} t$

where $C_{0}$ is the wave speed.

With a regular discretisation grid, the governing equations (eqn (6)) with the boundary conditions are numerically integrated.

\section{SHPB test simulation}

Using the presented numerical technique, fictitious tests can be simulated. However, it is more convincing to simulate a fictitious test close to a real one. Indeed, on applying the velocities measured in a real test, corresponding simulated forces can be calculated with any arbitrary set of parameters of the model. The comparison between simulated forces and measured ones allows for an adjustment of the

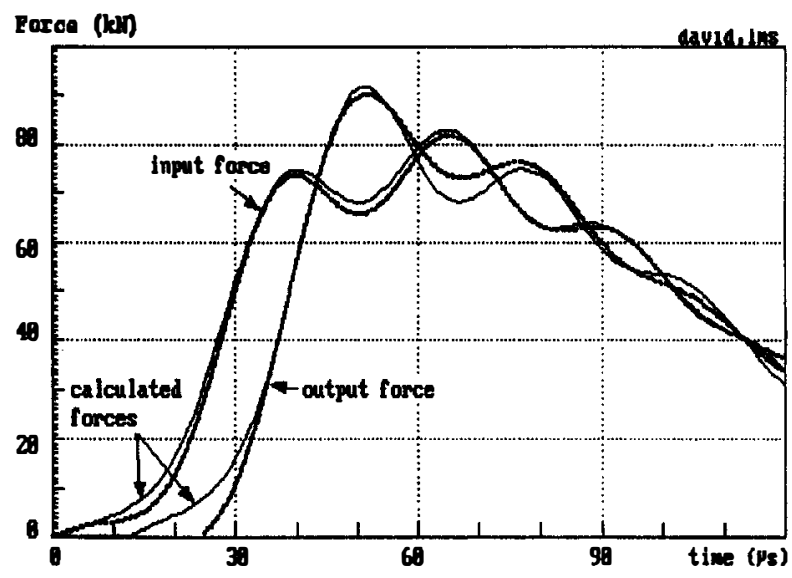

Fig. 6. Comparison between simulated and measured forces (SHPB).

parameters to obtain an optimised matching between them.

For instance, a real concrete test is performed with aluminium bars (length $2 \mathrm{~m}$, diameter $40 \mathrm{~mm}$ ). The specimen is a microconcrete one ( $36 \mathrm{~mm}$ diameter, $36 \mathrm{~mm}$ length). An excellent matching is found (Fig. 6) for this test with an optimised set of parameters $\quad\left(E=2 \times 10^{10} \mathrm{~Pa}, \quad k=1.5 \times 10^{10} \mathrm{~Pa}\right.$, $\left.\eta=8.5 \times 10^{5} \mathrm{~Pa} . \mathrm{s}\right)$.

It is then supposed that the simulated data gives a reasonable representation of this real test. In this way, one has the complete data of an idealised test where not only the three basic waves as in a real test, but also the specimen behaviour are known. The average stress-strain curve is then calculated from the waves with the classical formula (eqn (3)). Its comparison with the stress-strain curve given by the imposed model at the same strain rate history shows that this classical formula does not give a good estimate (Fig. 7).

However, another possible average formula ${ }^{24}$ (eqn (7)) has been used in the literature, which consists of calculating the stress by the average of the input and output forces.

$$
\begin{aligned}
& \dot{\varepsilon}_{\mathrm{s}}(t)=\frac{\mathrm{V}_{\text {output }}(t)-\mathrm{V}_{\text {input }}(t)}{l_{\mathrm{s}}}, \\
& \sigma_{\mathrm{s}}(t)=\frac{\mathrm{F}_{\text {input }}(t)+\mathrm{F}_{\text {output }}(t)}{2 S_{\mathrm{s}}}
\end{aligned}
$$

Fortunately, this formula can offer an acceptable result of the stress-strain relation (Fig. 7), 


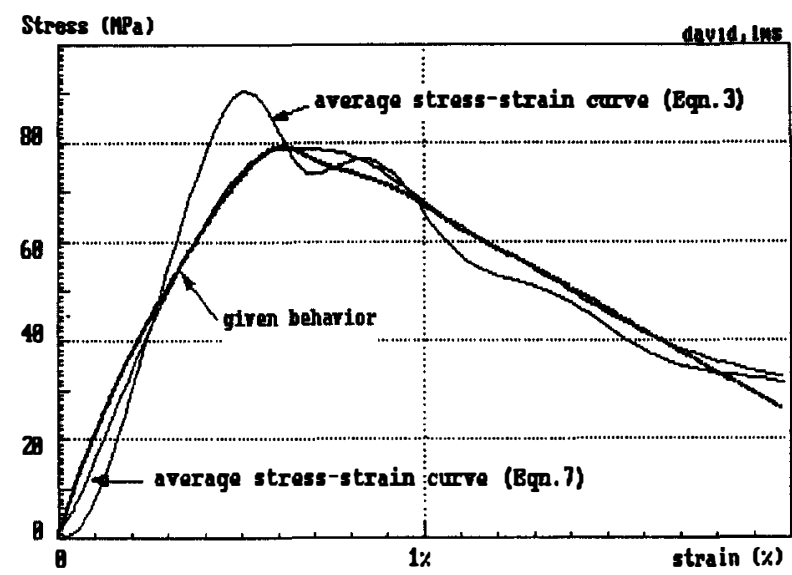

Fig. 7. Average stress-strain curves compared with the given behaviour (SHPB).

and it should then be used in the analysis of tests on concrete-like materials.

\section{DIHB test simulation and discussions, inverse approach}

In DIHB tests, velocity and force at the input side are obtained with supplementary assumptions because of the elimination of the input bar. Therefore, the quality of the formula eqn (7) strongly depends on those assumptions which are not analysed in the literature, probably because one has no evident means to obtain an experimental measure to check them.

One tries here to investigate those assumptions by simulation. Indeed, a fictitious test can be simulated with the imposed boundary conditions as the force and velocity measured at the output side in a real DIHB test, knowing that the integration of the Cauchy problem by the method of characteristics is also stable. ${ }^{25}$ If the simulation is performed with the realistic concrete-like model, the simulated test is supposed to be representative for a real DIHB test on a concrete specimen. Therefore, the force and the velocity at the input side are known in this realistic fictitious test. Comparisons between the simulated data at input side and those assumed by conventional DIHB analysis provide an estimate of the accuracy of DIHB supplementary assumptions.

The measured force and velocity at output side for the same kind of microconcrete specimen as previously used are obtained in a DIHP system (40 mm diameter aluminium output bar, $100 \mathrm{~mm}$ diameter steel projectile) with an initial projectile velocity of $7 \mathrm{~m} / \mathrm{s}$. The same model and parameter as those used in the previous simulation are applied to simulate input data.

It is shown in Fig. 8 that both the simulated force and the simulated velocity are quite different from the assumed values, which could not be due only to an eventually improper constitutive model. Consequently, the usual DIHB assumptions are not reliable for concrete-like materials, especially the assumption of the initial input velocity jump, because this infinitely small rise time implies an important error on the strain and the strain rate.

One has to know the rise time for the input velocity to obtain an acceptable accuracy. However, there is no evident measuring method and an assumption of an arbitrary rise time is not precise because it depends on the uniformity of contact between projectile and specimen at the instant of contact. ${ }^{9}$

Nevertheless, it is possible to develop a more accurate analysis by an inverse approach such as those proposed for SHPB. ${ }^{16}$ Indeed, an inverse

(a)

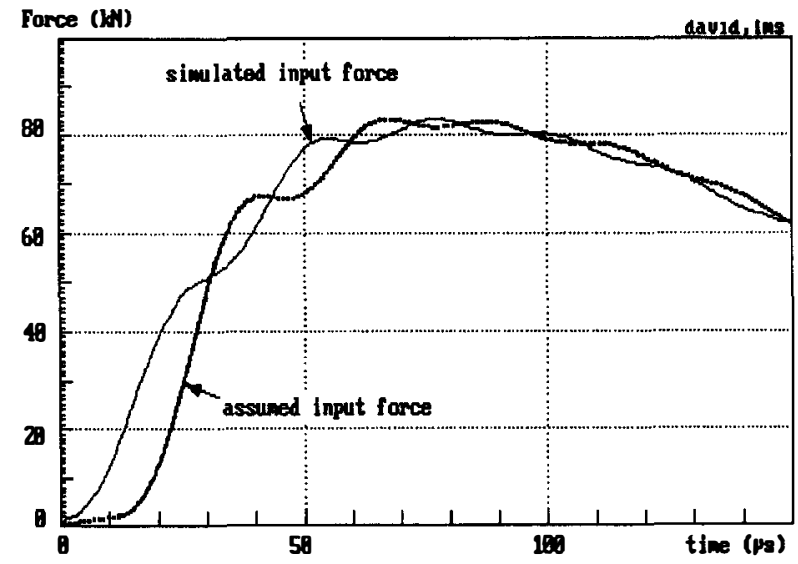

(b)

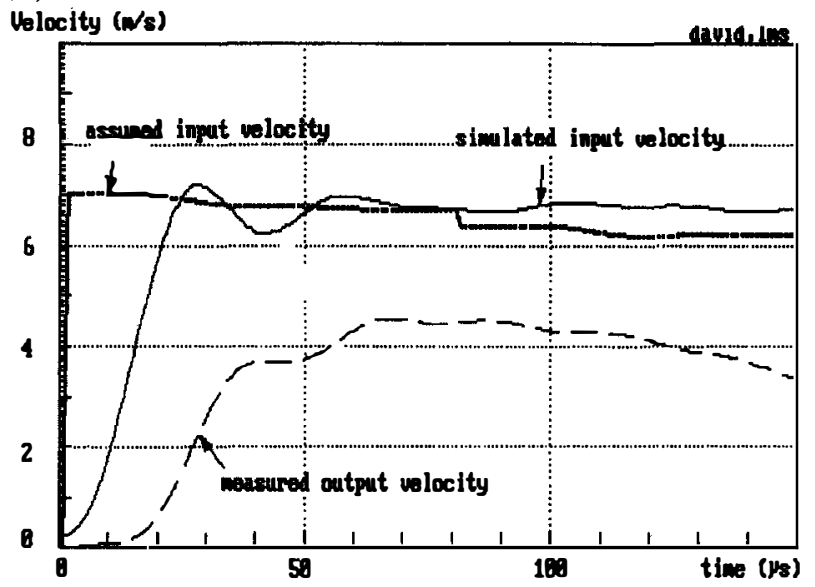

Fig. 8. (a) Comparison between simulated and assumed data (DIHB), input force. (b) Comparison between simulated and assumed data (DIHB), input velocity. 
method is possible when the superabundant data exists. In Fig. $8 \mathrm{~b}$, the assumed velocity after initial rise is reasonable and it is superabundant information. If an appropriate form of the material behaviour is supposed known with some parameters to be determined, input velocity can be simulated for a given set of parameters from the measured data at the output side. The comparison between the simulated input velocity and the assumed one for the period after initial rise offers a possibility to identify the optimal set of parameters.

\section{CONCLUSIONS}

In this paper, the accuracy of tests at high strain rate on concrete-like material is investigated. On the one hand, the shifting of waves, which are the only directly measured data, will not introduce significant error provided that the wave dispersion correction and exact time shift are carefully taken into account. On the other hand, a transient numerical simulation is developed using a method of characteristics and a realistic crack model. For the SHPB test, this simulation permits for the validation of stressstrain relation given by one of the conventional analyses. For the DIHB test, it is shown by the simulation that the usual assumption is not accurate and an inverse approach can probably offer more accurate results.

\section{REFERENCES}

1. Green H., The impact testing of concrete. In Walton, W. H. (ed.), Mechanical Properties of Non-metallic Brittle Materials, 1958, pp. 300-317.

2. RILEM committee. The effect of impact loading on building. State-of-art report. Matériaux et Constructions, 8 (1975) 77-125.

3. Gong, J. C., Malvern, L. E. \& Jenkins, D. A., Dispersion investigation in the split Hopkinson pressure bar. J. Engng. Mater. Tech., 112 (1990) 309-314.

4. Tang, T., Malvern, L. E. \& Jenkins, D. A., Rate effects in uniaxial dynamic compression of concrete. J. Engng. Mech., 118 (1992) 108-124.

5. Reinhardt, H. W., Körmeling, H. A. \& Zielinski, A. J., The split Hopkinson bar, a versatile tool for the impact testing of concrete. Matériaux et Constructions, 19 (1986) 55-63.

6. Gary G., Klepaczko, J. R., Résumé des résultats expérimentaux sur mini-béton, essai de compression. Rapport scientifique GRECO (1992) 105-119.
7. Zhao, H. \& Gary, G., A new method for the separation of waves. Application to the SHPB technique for an unlimited measuring duration. J. Mech. Phys. Solids, 45 (1997) 1185-1202.

8. Kolsky, H., An investigation of mechanical properties of materials at very high rates of loading. Proc. Phys. Soc. London, Series B, 62 (1949) 676-700.

9. Dharan, C. K. H. \& Hauser, F. E., Determination of stress-strain characteristics at very high strain rates. Exp. Mech., 10 (1970) 370-376.

10. Pope, P. H. \& Field, J. E., Determination of strain in a dynamic compression test. J. Phys. E: Sci. Intrum., 17 (1984) 817-820.

11. Walley, S. M., Field, J. E., Pope, P. H. \& Safford, N. A., The rapid deformation behavior of various polymer. J. Phys. III, 1 (1991) 1889-1925.

12. Gorham, D. A., Pope, P. H. \& Field, J. E., An improved method for compressive stress-strain measurements at very high strain rates. Proc. $R$. Soc. Lond. A, 438 (1992) 153-170.

13. Davies, R. M., A critical study of Hopkinson pressure bar. Phil. Trans. Roy. Soc. A, 240 (1948) 375-457.

14. Follansbee, P. S. \& Franz, C., Wave propagation in the split Hopkinson pressure bar. J. Engng. Mater. Tech., 105 (1983) 61-66.

15. Zhao, H. \& Gary, G., A three-dimensional analytical solution of longitudinal wave propagation in an infinite linear viscoelastic cylindrical bar. Application to experimental techniques. J. Mech. Phys. Solids., 43 (1995) 1335-1348.

16. Zhao, H. \& Gary, G., On the use of SHPB techniques to determine the dynamic behavior of materials in the range of small strains. Int. J. Solids and Structures, 33 (1996) 3363-3375.

17. Ravichandran, G. \& Subhash, G., Critical appraisal of limiting strain rate for compression testing of ceramics in a split Hopkinson pressure bar. J. American Ceramic Society, 77 (1994) 263-267.

18. Zhao, H. \& Gary, G., An experimental investigation of compressive failure strength of fibre polymer matrix composite plates under impact loading. Composite Sci. and Technol., 57 (1997) 287-292.

19. Davies, E. D. H. \& Hunter, S. C., The dynamic compres]sion testing of solids by the method of the split Hopkinson pressure bar. J. Mech. Phys. Solids, 11 (1963) 155-179.

20. Bertholf, L. D. \& Karnes, J., Two-dimensional analysis of the split Hopkinson pressure bar system. $J$. Mech. Phys. Solids, 23 (1975) 1-19.

21. Malinowski, J. Z. \& Klepaczko, J. R., A unified analytic and numerical approach to specimen behavior in the split Hopkinson pressure bar. Int. J. Mech. Sci., 28 (1986) 381-391.

22. Sluys, L. J. \& de Borst, R., Wave propagation and localisation in rate-dependant cracked medium-Model formulation and one-dimensional example. Int. J. Solids and Struct., 29 (1992) 2945-2958.

23. Zhao, H., A study of specimen thickness effects in the impact tests on polymers by numeric simulations. Polymer, in press.

24. Lindholm, U. S., Some experiments with the split Hopkinson pressure bar. J. Mech. Phys. Solids, 12 (1964) 317-335.

25. Rota, L., Application de méthode inverse au dépouillement de l'essai aux barres de Hopkinson. Ph.D. Thesis, Ecole Polytechnique, Paris, 1997. 